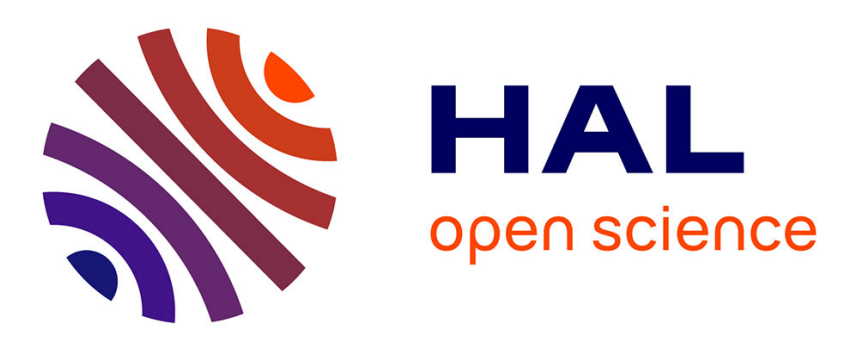

\title{
Gas-phase conformations of 2-methyl-1,3-dithiolane investigated by microwave spectroscopy
} Vinh Van, Wolfgang Stahl, Martin Schwell, Ha Vinh Lam Nguyen

\section{To cite this version:}

Vinh Van, Wolfgang Stahl, Martin Schwell, Ha Vinh Lam Nguyen. Gas-phase conformations of 2methyl-1,3-dithiolane investigated by microwave spectroscopy. Journal of Molecular Structure, In press, 1156, pp.348-352. 10.1016/j.molstruc.2017.11.084 . hal-03183073

\section{HAL Id: hal-03183073 https://hal.science/hal-03183073}

Submitted on 26 Mar 2021

HAL is a multi-disciplinary open access archive for the deposit and dissemination of scientific research documents, whether they are published or not. The documents may come from teaching and research institutions in France or abroad, or from public or private research centers.
L'archive ouverte pluridisciplinaire HAL, est destinée au dépôt et à la diffusion de documents scientifiques de niveau recherche, publiés ou non, émanant des établissements d'enseignement et de recherche français ou étrangers, des laboratoires publics ou privés. 


\title{
Gas-phase conformations of 2-methyl-1,3-dithiolane investigated by microwave spectroscopy
}

\author{
Vinh Van ${ }^{\text {a }}$, Wolfgang Stahl ${ }^{\text {a }}$, Martin Schwell ${ }^{\text {, }}$, Ha Vinh Lam Nguyen ${ }^{\text {b,* }}$ \\ ${ }^{a}$ Institute of Physical Chemistry, RWTH Aachen University, Landoltweg 2, D-52074 Aachen, \\ Germany; ${ }^{b}$ Laboratoire Interuniversitaire des Systèmes Atmosphériques (LISA), CNRS UMR 7583, \\ Université Paris-Est Créteil, Université Paris Diderot, 61 avenue du Général de Gaulle, F-94010 Créteil \\ cedex, France \\ * Corresponding author: lam.nguyen@lisa.u-pec.fr
}

\begin{abstract}
The conformational analysis of 2-methyl-1,3-dithiolane using quantum chemical calculations at some levels of theory yielded only one stable conformer with envelope geometry. However, other levels of theory indicated two envelope conformers. Analysis of the microwave spectrum recorded using two molecular jet Fourier transform microwave spectrometers covering the frequency range from 2 to $40.0 \mathrm{GHz}$ confirms that only one conformer exists under jet conditions. The experimental spectrum was reproduced using a rigid-rotor model with centrifugal distortion correction within the measurement accuracy of $1.5 \mathrm{kHz}$, and molecular parameters were determined with very high accuracy. The gas phase structure of the title molecule is compared with the structures of other related molecules studied under the same experimental conditions.
\end{abstract}

Keywords: rotational spectroscopy $\bullet$ conformational analysis $\bullet$ dithiolane $\bullet$ five-membered ring - ab initio calculations 


\section{Introduction}

Volatile sulfur-containing heterocyclic molecules are important flavor substances and raw materials for perfumes. It is known that sulfur-containing odorants of the chemical classes mercaptan (R-SH) and thioether (R-S-R') are intensely bad-smelling, especially in high concentrations, while the oxygen analogs, alcohol (R-OH) and ether (R-O-R'), have usually less perceptible smell or in many cases a much more pleasant odor. The reason for this phenomenon has not yet been completely understood. An explanation by Fischer that the sulfurcontaining substances are recognized by human smelling receptors by the "lock and key" model [1] is not satisfactory, since almost no remarkable changes of the molecular structures could be found between the oxygen and the sulfur-containing analogs. Furthermore, the typical odors of sulfur-containing molecules cannot be imitated by sulfur-free substances which have similar molecular geometry and charge distribution. On the other hand, hydrogen bonds formed by sulfur-containing substances are much weaker than those by molecules with oxygen [2]. Therefore, we suppose that sulfur-containing substances form reversible bonds directly between the sulfur atom with sulfur-containing amino acids in the proteins in the human receptors, which is not possible in the respective oxygen analogues. To understand and explain this phenomenon in more details, it is important to answer the questions how molecular shapes fit together. For this purpose, gas phase structures of sulfur-containing odorants are needed, since the sense of smell starts from gas phase molecules. A huge number of oxygen containing odorants were investigated in the gas phase, including the studies on linalool [3], a main component of lavender oil, on fruit esters such as isoamyl acetate (banana odor) [4], ethyl valerate (green apple) [5] as well as on cassyrane (blackberry) [6]. Considerably fewer gas phase investigations on sulfur-containing odorants have been carried out, though they are often the odor compounds found in nature. Some examples are cat ketone [7], dihydro-2-methyl-3-thiophenone [8], and methyl isopropyl sulfide [9]. 
Here, we report on the gas phase structure of 2-methyl-1,3-dithiolane (MDT, see Fig. 1), a volatile five-membered ring which contains two sulfur atoms, obtained using a combination of quantum chemical calculations and Fourier transform microwave (FTWM) spectroscopy in a supersonic jet. This technique is ideally suited to determine the structures of sizeable molecules where different stable conformers exist whose energy differences are small and conformational distinction is not always possible by quantum chemical calculations alone. The results presented can be used for structure-activity correlation studies, as well as for benchmarks to improve theoretical models, especially because there is still significant interest in characterizing the various conformers of organic molecules in terms of relative energies, structures, and dipole moments. Finally, the molecular geometry of MDT will be compared with the structures of other related five-membered rings.

\section{Quantum Chemical Calculations}

MDT is a five-membered heterocyclic molecule with two sulfur atoms at the 1 and 3 positions of the ring and a methyl group attached to the carbon at the 2 position. At the beginning, several starting geometries were created based on the structures of the two conformers of cyclopentane [10]. Geometry optimizations were carried out at the MP2/6-311++G(d,p) level of theory using the GAUSSIAN09 package [11] to search for stable conformers. Quantum chemistry is a powerful tool to understand microwave spectra. The optimized geometries and molecular parameters of stable conformers give reasonable estimations to start the spectral assignment. Conversely, experimental parameters obtained with high accuracy by microwave spectroscopy can be used for benchmark calculations. For example, in the study of Grimme et al. [12] the experimental data of isoamyl acetate [4], diisopropyl ketone [13], and triethyl amine [14] were used to validate the quantum chemical results.

The MP2/6-311++G(d,p) level of theory was chosen, because it yields rotational constants which are in close agreement with the experimental values for other five-membered 
rings $[15,16]$. Anharmonic frequency calculations were carried out at the same level of theory to check, whether the optimized geometries are true minima or saddle points, and to obtain centrifugal distortion constants.

We found only one conformer which has an envelope structure as shown in Fig. 1. The calculated rotational constants $A=2.780, B=2.724$, and $C=1.605 \mathrm{GHz}$ indicate that MDT is a near oblate top with a Ray's asymmetry parameter $\kappa=0.903$. The Cartesian coordinates of this conformer are given in Table S-1 in the Electronic Supporting Information (ESI). This is in contrary to many previous investigations on other five-membered rings where structure optimizations delivered two conformers (see Section 5 which follows). Therefore, we repeated the calculations at different levels of theory using various combinations of the HF, DFT, MP2, and CCSD methods and different basis sets including correlation consistent and Pople basis sets [17] to check for convergence. Surprisingly, some levels of theory yielded two optimized conformers (see Table S-2 in the ESI). Their geometries depend on the level of theory in use, but in most cases they have structures which are mixtures of twist and envelope. Harmonic frequency calculations were performed for all optimized structures, which confirmed that almost all of them are stable minima. Interestingly, the second conformer obtained at two levels using the M06-2X method shows one imaginary frequency, meaning that it is a saddle point and not a stable conformer. If not else stated, all values given from now on will refer to the MP2/6-311++G(d,p) level of theory.

MDT features a methyl group which, in general, exhibits internal rotation and causes A-E splittings of all rotational lines in the microwave spectrum [18]. We calculated the barrier to internal rotation and obtained a value of $1650 \mathrm{~cm}^{-1}$. Sample one-top calculations using the program XIAM [19] show that torsional splittings are small and cannot be resolved with the resolution of our spectrometers. This is not surprising, because in many other molecules 
where $n$-alkyl methyl group is present [20-22], the A-E splittings were not observable under the same measurement conditions.
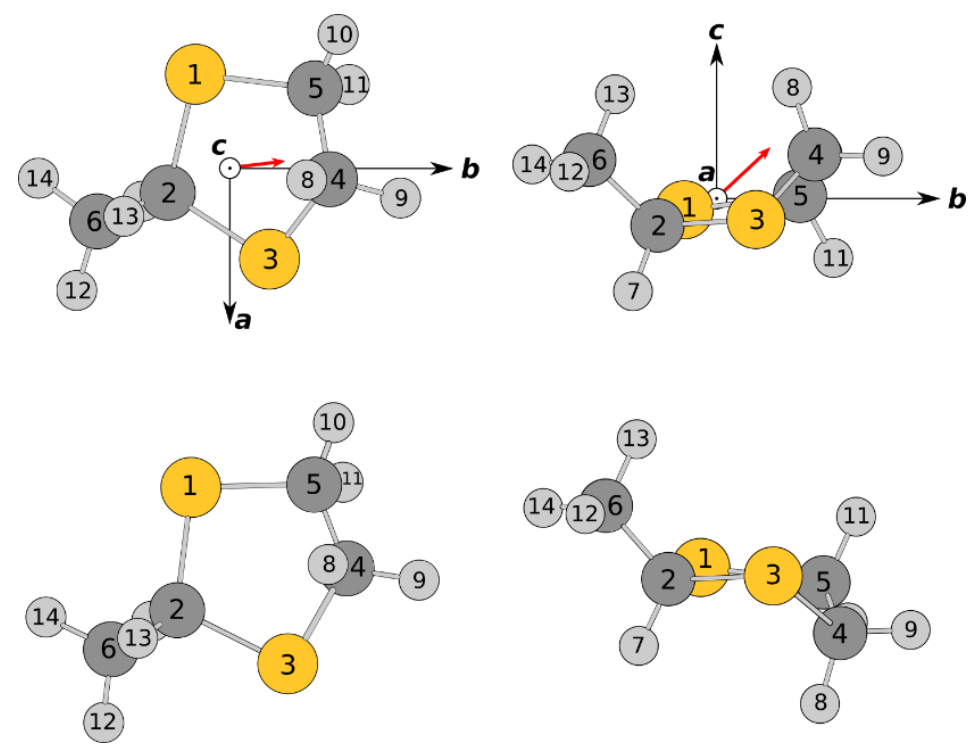

Fig. 1. Optimized geometries of 2-methyl-1,3-dithiolane. Upper trace: the only stable conformer obtained at all levels of theory in its principal axis system. Left hand side: view along the $c$-principal axis; right hand side: view along the $a$-principal axis. The circled dots indicate that the axis direction points out of the paper plane. The red arrow represents the dipole moment vector. Lower trace: the second conformer of 2-methyl-1,3-dithiolane obtained only at some levels of theory. The geometry calculated at the MP2/cc-pVTZ is illustrated in complementary views to those in the upper trace. The energy is $0.89 \mathrm{~kJ} \cdot \mathrm{mol}^{-1}$ higher than that of the first conformer.

\section{Microwave Spectroscopy}

\subsection{Measurements}

The rotational spectra were recorded with two molecular jet FTMW spectrometers operating in the frequency range from 2 to $40 \mathrm{GHz}$. The spectrometer located in Aachen, Germany, operates from $2-26.5 \mathrm{GHz}$ [23] and the other one in Paris, France, subsequently from $26.5-$ $40 \mathrm{GHz}$ [24]. Details of the spectrometers are given in the literature and will not be repeated here. MDT was purchased from Alfa Aesar GmbH \& Co. KG, Karlsruhe, Germany. The stated 
purity was $99 \%$ and we used the sample without further purification. A piece of pipe cleaner was used as sample carrier and was soaked with the liquid substance, then placed in front of the nozzle of the spectrometers. For the measurements, a helium stream at a pressure of approximately $200 \mathrm{kPa}$ was used. The helium-MDT mixture was expanded through the pulsed nozzle into the cavity. Helium was chosen as carrier gas instead of argon or neon since the molecular beam is warmer and higher energy levels are still populated. This is important for obtaining accurate centrifugal distortion constants.

A broadband scan was recorded in the frequency range from 9 to $14 \mathrm{GHz}$, where overlapping spectra were automatically taken with a step width of $0.25 \mathrm{MHz}$. Afterwards, all lines were remeasured in the high resolution mode of the spectrometers, where they appeared as doublets due to the Doppler effect. The mean value of the line widths is approximately 15 $\mathrm{kHz}$. The measurement accuracy of isolated lines is estimated to be $1.5 \mathrm{kHz}$. A portion of the scan as well as a typical high resolution spectrum is illustrated in Fig. 2.

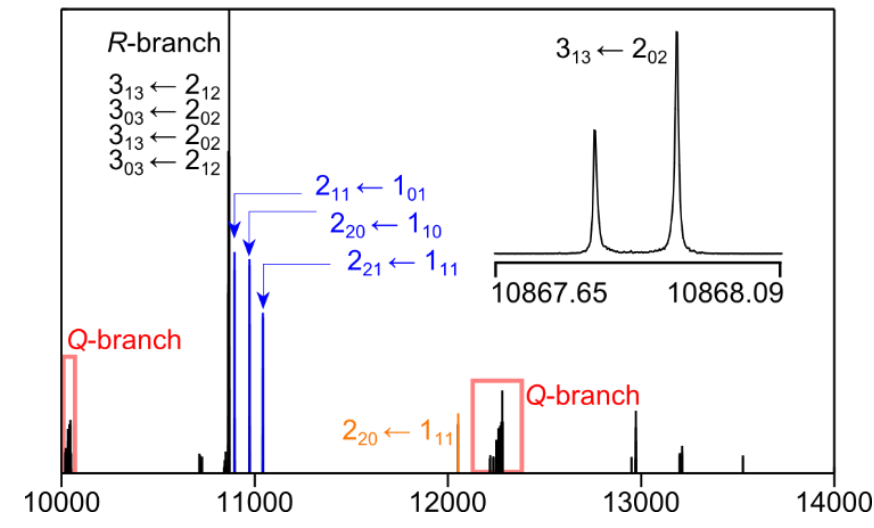

Fig. 2. A portion of the broadband scan of 2-methyl-1,3-dithiolane (power spectrum) in the frequency range from 10000 to $14000 \mathrm{MHz}$. Three $c$-type $R$-branch transitions $2_{11} \leftarrow 1_{01}, 2_{20} \leftarrow 1_{10}$, and $2_{21} \leftarrow 1_{11}$ are marked in blue and an isolated $b$-type $R$-branch transition $2_{20} \leftarrow 1_{11}$ in orange. The overlapping $a$ and $b$-type $Q$ - and $R$-branches are also indicated. A typical high resolution spectrum of the $3_{13} \leftarrow 202$ transition is illustrated in the upper right corner. 


\subsection{Spectral Assignment}

The microwave spectrum of the only conformer of MDT optimized at the MP2/6-311++G(d,p) level of theory was predicted with the program XIAM in its rigid-rotor mode using the rotational constants given in Section 2.1. The dipole moment components $\left|\mu_{\mathrm{a}}\right|=0.12 \mathrm{D},\left|\mu_{\mathrm{b}}\right|=0.80 \mathrm{D}$, and $\left|\mu_{c}\right|=0.80 \mathrm{D}$ suggest a spectrum with intense $b$ - and $c$-type and weaker $a$-type transitions. The predicted spectrum shows that the frequencies of all $a$ - and $b$-type $Q$ - and $R$-branches present in the broadband scan region are very narrow. Those transitions are almost overlapped. This is also our observation in the experimental spectrum, where some frequency regions with a high line density can be recognized (see Fig. 2). Therefore, we started our assignment with the clearly separated $c$-type transitions $2_{11} \leftarrow 1_{01}, 2_{20} \leftarrow 1_{10}$, and $2_{21} \leftarrow 1_{11}$ and an isolated $b$-type transition $2_{20} \leftarrow 1_{11}$ as shown in Fig. 2 . These lines could be assigned straightforward, which enabled us to find further separated lines, first in the broadband scan region, then over the whole frequency range up to $40 \mathrm{GHz}$. Finally, the highly dense frequency range with overlapping $a$ - and $b$-type transitions mentioned above could also be assigned, after the rotational and centrifugal distortion constants were improved. The fitted molecular parameters are summarized in Table 1, where they are compared with the calculated values. All fitted transitions are listed in Table S-3 in the ESI.

\section{Discussion}

In total, 136 transitions are fitted to an excellent standard deviation below $1.5 \mathrm{kHz}$, which is within the measurement accuracy. The rotational and centrifugal distortion constants are determined with very high accuracy. The experimentally deduced rotational constants were compared with those from quantum chemical calculations. Except for calculations at the HF levels, the predicted rotational constants are in good agreement to the experimental values (see Table S-2), despite the fact that quantum chemical data refer to the equilibrium structure, whereas the experimental data yield rotational constants for the ground vibrational state and no 
corrections have been made. Only at the MP2/6-311++G(d,p) level of theory, we carried out anharmonic frequency calculations to provide the ground state $r_{0}$ structure. However, the $B_{0}$ rotational constants given in Table 1 do not always agree better with the experimental values than the $B_{\mathrm{e}}$ values obtained at different levels of theory listed in Table S-2.

The barrier to internal rotation of the methyl group is calculated to be higher than $1650 \mathrm{~cm}^{-1}$ and trial one-top calculations using the program XIAM indicates very small torsional splittings. In agreement to this prediction, all lines appear sharp and no torsional splittings are observed. Finally, we mention that all rotational transitions present in the broadband scan are assigned to one conformer, clearly indicating that only one conformer is present under our molecular jet conditions. These experimental results strongly support calculations at those levels of theory given in Table S-2 which yielded only one stable conformer for MDT. Depending on the dipole moment, the vapor pressure of the substance, and its partition function, in some of our previous studies on sulfur-containing molecules it was also possible to detect ${ }^{34} \mathrm{~S}$ isotopologues in its natural abundance of $4 \%$ in the broadband scan $[8,15]$. However, in the cases of MDT and also other molecules [25] the overall intensity was too small to detect them.

\section{Comparison to related molecules}

In this section, the structure of the observed conformer of MDT will be compared to those of other five-membered rings shown in Fig. 3. A recent study under the same experimental conditions on the gas phase structures of 2-methyltetrahydrothiophene (1), where one sulfur atom is substituted with a methylene group in comparison to MDT, reported that (1) exists in

form of two stable twist conformers, called conformer I and II in Ref. (15) and conformer twist 1-I and twist 1-II, respectively, in the present work (visualized in Fig. 4). 


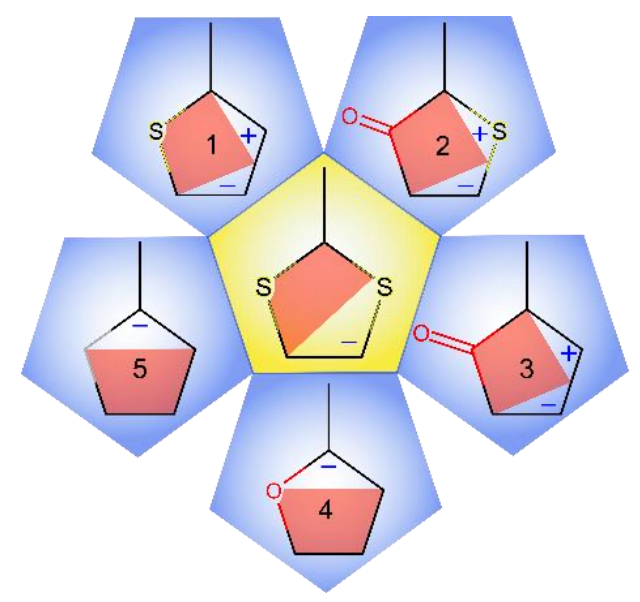

Fig. 3. Molecules related to 2-methyl-1,3-dithiolane: (1) 2-methyltetrahydrothiophene, (2) dihydro-2methyl-3-thiophenone, (3) 2-methylcyclo-pentanone, (4) 2-methyltetrahydrofuran, and methylcyclopentane. Red quadrilaterals indicate the ring planes. Surfaces marked by $(+)$ and $(-)$ are in front of and behind the ring planes, respectively.

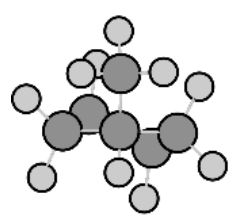

twist 5-1

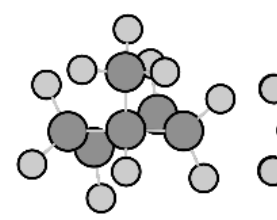

twist 5-I*<smiles>OC1OC2OOC1O2</smiles>

envelope 5-I

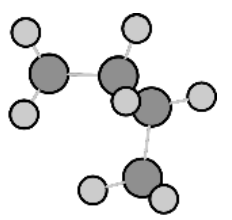

envelope $\mathbf{5}$-II
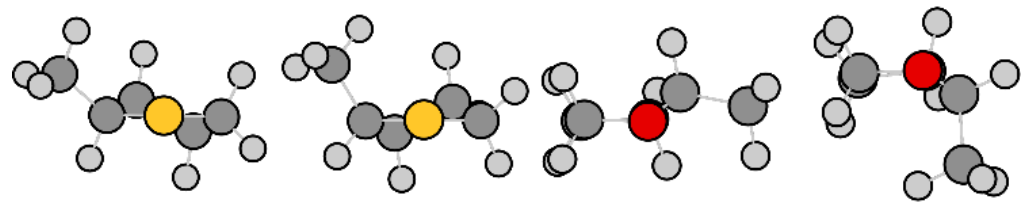

twist 1-I

twist 1-II

envelope 4-I

envelope 4-II
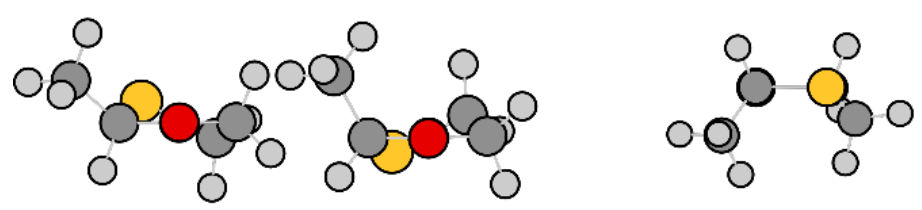

MDT

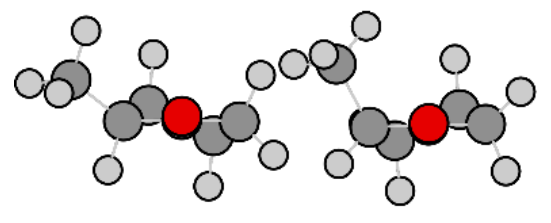

twist 3-I

twist 3-II

Fig. 4. Comparison of the experimental structures of six molecules shown in Fig. 3. For conformer names, see text. 
If a sulfur atom is exchanged by a carbonyl group, we obtain dihydro-2-methyl-3thiophenone (2) from MDT. In a microwave spectroscopic study, also under the same experimental conditions, Mouhib et al. indicated two stable twist conformers, called conformer I and II in Ref. (8) and conformer twist 2-I and twist 2-II, respectively, in Fig. 4. In those structures, the carbonyl group clearly dominates the thioether group, which now acts like a normal methylen group in the ring. This statement is supported by comparing these gas phase geometries of (2) with those of 2-methylcyclopentanone (3) [26]. Also in this molecule, two stable twist conformers called equatorial and axial conformers in Ref. (26) and conformer twist 3-I and twist 3-II, respectively, in Fig. 4 are noted. Only the more stable equatorial conformer was found in the experimental spectrum.

We found only twist conformers for molecules (1), (2), and (3). In all these cases, the lowest energy conformer, twist $\mathbf{n}-\mathrm{I}(\mathbf{n}=\mathbf{1 - 3})$, has the methyl group and the neighboring methylene group on the same side of the $\mathrm{C}-\mathrm{S}-\mathrm{C}$ or $\mathrm{C}-(\mathrm{C}=\mathrm{O})-\mathrm{C}$ plane. In contrast, if the ether group is present in the ring instead of the thioether and/or the carbonyl group, as in the case of methyltetrahydrofurane (4), only stable envelope conformers are obtained, as reported in Ref. (16). Measurements of (4) were carried out under the same experimental conditions, which enable comparable results. These conformers are called equatorial and axial conformers in Ref. (16), but envelope 4-I and envelope 4-II, respectively, in Fig. 4.

If we remove the functional groups in MDT, (1), (2), (3), and (4), we obtain the prototype molecule methylcyclopentane (5). No microwave spectroscopic studies are available for this molecule, probably because of its low dipole moment (about $0.1 \mathrm{D}$, see Table S- $4 \mathrm{~h}$ and S-4i). Quantum chemical calculations at the MP2/6-311++G(d,p) level yield in total four conformers: two envelope, called envelope 5-I and envelope 5-II, and two twist conformers, called twist 5-I and twist 5-I* (enantiomers) in Fig. 4. The structures of the envelope conformers are very similar to those of conformers envelope 4-I and envelope 4-II of 
methyltetrahydrofurane, and the structures of the twist conformers to those of conformers twist $\mathbf{n}$-I and twist $\mathbf{n}$-II $(\mathbf{n}=\mathbf{1 - 3})$. The Cartesian coordinates of all conformers given in Fig. 4 are summarized in Table S-4 in the ESI.

In summary, while the prototype molecule methylcyclopentane has two stable twist and two stable envelope conformers, the envelope conformers disappear if a thioether and/or a carbonyl group is present in the ring. If an ether group is present, as in the case of methyltetrahydrofurane, the twist conformers disappear and only the envelope structures are found. MDT shows itself as a unique case in the five-membered heterocyclic family, because only one envelope structure is present and the envelope flap is at a different position than in methyltetrahydrofurane (4) and methylcyclopentane (5) (see Figs. 3 and 4). In both (4) and (5), the methyl group is attached on the top of the envelope flap. In MDT, it is attached on the carbon atom located in the ring plane. Currently, we do not have a good explanation for this observation. Gas phase structures of further related molecules, for example 2-methyl-1,3dioxolane, might help at least to state whether this is due to a sterical effect.

\section{Acknowledgements}

V.V. thanks the Fonds der Chemischen Industrie (FCI) for a Ph.D. fellowship. Simulations were performed with computing resources granted by JARA-HPC from the RWTH Aachen University under the project jara0124.

\section{References}

[1] E. Fischer, Berichte der Deutschen chemischen Gesellschaft zu Berlin 27 (1894) 2985-2993.

[2] L. M. Gregoret, S. D. Rader, R. J. Fletterick, F. E. Cohen, Proteins 9 (1991) 99-107. 
[3] H. V. L. Nguyen, H. Mouhib, S. Klahm, W. Stahl, I. Kleiner, Phys. Chem. Chem. Phys. 15 (2013) 10012-10018.

[4] L. W. Sutikdja, D. Jelisavac, W. Stahl, I. Kleiner, Mol. Phys. 110 (2012) 2883-2893.

[5] H. Mouhib, W. Stahl, Chem. Phys. Chem. 13 (2012) 1297-1301.

[6] H. Mouhib, W. Stahl, M. Lüthy, M. Büchel, P. Kraft, Angew. Chem. Int. Ed. 50 (2011) 55765580.

[7] H. Mouhib, W. Stahl, Chem. Biodivers. 11 (2014) 1554-1566.

[8] H. Mouhib, V. Van, W. Stahl, J. Phys. Chem. A 117 (2013) 6652-6656.

[9] E. Hirota, K. Sakieda, Y. Kawashima, Phys. Chem. Chem. Phys. 12 (2010) 8398-8404.

[10] D. A. Lightner, J. E. Gurst, Organic Conformational Analysis and Stereochemistry from Circular Dichroism Spectroscopy, J. Wiley-VCH, New York, 2000.

[11] M. J. Frisch, G. W. Trucks, H. B. Schlegel, G. E. Scuseria, M. A. Robb, J. R. Cheeseman, G. Scalmani, V. Barone, B. Mennucci, G. A. Petersson, H. Nakatsuji, M. Caricato, X. Li, H. P. Hratchian, A. F. Izmaylov, J. Bloino, G. Zheng, J. L. Sonnenberg, M. Hada, M. Ehara, K. Toyota, R. Fukuda, J. Hasegawa, M. Ishida, T. Nakajima, Y. Honda, O. Kitao, H. Nakai, T. Vreven, J. A., Jr. Montgomery, J. E. Peralta, F. Ogliaro, M. Bearpark, J. J. Heyd, E. Brothers, K. N. Kudin, V. N. Staroverov, R. Kobayashi, J. Normand, K. Raghavachari, A. Rendell, J. C. Burant, S. S. Iyengar, J. Tomasi, M. Cossi, N. Rega, J. M. Millam, M. Klene, J. E. Knox, J. B. Cross, V. Bakken, C. Adamo, J. Jaramillo, R. Gomperts, R. E. Stratmann, O. Yazyev, A. J. Austin, R. Cammi, C. Pomelli, J. W. Ochterski, R. L. Martin, K. Morokuma, V. G. Zakrzewski, G. A. Voth, P. Salvador, J. J. Dannenberg, S. Dapprich, A. D. Daniels, O. Farkas, J. B. Foresman, J. V. Ortiz, J. Cioslowski, D. J. Fox, Gaussian 09, Revision A.02, Gaussian, Inc., Wallingford CT, 2009.

[12] S. Grimme, M. Steinmetz, Phys. Chem. Chem. Phys. 15 (2013) 16031-16042.

[13] Y. Zhao, H. Mouhib, W. Stahl, J. Phys. Chem. A 117 (2013) 311-314. 
[14] H. V. L. Nguyen, R. Kannengießer, W. Stahl, Phys. Chem. Chem. Phys. 14 (2012) 1175311758.

[15] V. Van, C. Dindic, W. Stahl, H.V.L. Nguyen, ChemPhysChem 16 (2015) 291-294.

[16] V. Van, W. Stahl, H.V.L. Nguyen, J. Mol. Struct. 1123 (2016) 24-29.

[17] R. Ditchfield, W. J. Hehre, J. A. Pople, J. Chem. Phys. 54 (1971) 724-728.

[18] H. Dreizler, Z. Naturforsch. 16a (1961) 1354-1367.

[19] H. Hartwig, H. Dreizler, Z. Naturforsch. 51a (1996) 923-932.

[20] R. Kannengießer, W. Stahl, H. V. L. Nguyen, J. Phys. Chem. A 120 (2016) 5979.

[21] L. Ferres, W. Stahl, H.V.L. Nguyen, Mol. Phys. 114 (2016) 2788-2793.

[22] A.O. Hernandez-Castillo, C. Abeysekera, B.M. Hays, I. Kleiner, H.V.L. Nguyen, T.S. Zwier, J. Mol. Spectrosc. 337 (2017) 51-58.

[23] J.-U. Grabow, W. Stahl, H. Dreizler, Rev. Sci. Instrum. 67 (1996) 4072-4084.

[24] I. Merke, W. Stahl, H. Dreizler, Z. Naturforsch. 49a (1994) 490-496.

[25] V. Van, W. Stahl, H. V. L. Nguyen, Phys. Chem. Chem. Phys. 17 (2015) 32111-32114.

[26] J. L. Alonso, J. C. López, R. M. Villamañán, J. Mol. Spectrosc. 126 (1987) 348-355. 
Table 1. Molecular parameters of the only observed conformer of 2-methyl-1,3-dithiolane obtained by the program XIAM in its rigid-rotor mode.

\begin{tabular}{llll}
\hline Par. $^{\mathrm{a}}$ & $\mathrm{Unit}$ & Experiment & $\mathrm{MP2}^{\mathrm{b}}$ \\
\hline$A$ & $\mathrm{GHz}$ & $2.77919037(14)$ & 2.761217 \\
$B$ & $\mathrm{GHz}$ & $2.705487270(73)$ & 2.697954 \\
$C$ & $\mathrm{GHz}$ & $1.624829803(60)$ & 1.598262 \\
$D_{J}$ & $\mathrm{kHz}$ & $0.5982(12)$ & 0.89643 \\
$D_{J K}$ & $\mathrm{kHz}$ & $-0.4194(55)$ & -2.3234 \\
$D_{K}$ & $\mathrm{kHz}$ & $0.4574(48)$ & 2.2468 \\
$d_{1}$ & $\mathrm{kHz}$ & $-0.15494(39)$ & -0.24605 \\
$d_{2}$ & $\mathrm{kHz}$ & $-0.14000(23)$ & -0.07182 \\
$\sigma^{\mathrm{c}}$ & $\mathrm{kHz}$ & 1.42 & \\
$\mathrm{~N}^{\mathrm{d}}$ & & 136 & \\
\hline
\end{tabular}

a All parameters are given with one standard uncertainty in parentheses. Watson's S reduction and Ir representation was used. ${ }^{\mathrm{b}}$ Vibrational ground state $B_{0}$ rotational constants and centrifugal distortion constants obtained by anharmonic frequency calculation at the MP2/6-311++G(d,p) level of theory. ${ }^{c}$ Standard deviation of the fit. ${ }^{\mathrm{d}}$ Number of lines. 